\title{
Predictors of weight loss and maintenance in patients treated with antiobesity drugs
}

\author{
This article was published in the following Dove Press journal: \\ Diabetes, Metabolic Syndrome and Obesity:Targets and Therapy \\ 17 June 2011 \\ Number of times this article has been viewed
}

\author{
Federica Guaraldi' \\ Uberto Pagotto ${ }^{2}$ \\ Renato Pasquali² \\ 'Department of Pathology, \\ The Johns Hopkins University \\ School of Medicine, Baltimore, MD, \\ USA; ${ }^{2}$ Division of Endocrinology, \\ Department of Clinical Medicine, \\ S Orsola-Malpighi Hospital, Alma \\ Mater Studiorum University, \\ Bologna, Italy
}

\begin{abstract}
Background: The prevalence of obesity and related diseases has increased enormously in the last few decades, becoming a very important medical and social issue. Because of the increasing number of people who need weight loss therapies and the high costs associated with these, the search for reliable predictors of success for weight loss and weight maintenance treatments has become a priority.

Objective: A literature review was undertaken to identify possible predictors of outcome of weight loss and weight maintenance in patients treated with antiobesity drugs.

Results: For the majority of variables, published data are not sufficient to define their role on final outcomes. Among all considered factors, only early response to treatment appeared to be a reliable positive predictor, and diabetes a negative predictor of weight loss and maintenance.

Conclusion: To date, no definitive results have been obtained. Due to the great benefits of reliable predictors of outcome associated to currently available antiobesity drugs and those under development, identifying these predictors has to be supported and encouraged.
\end{abstract}

Keywords: obesity, weight loss predictors, pharmacological treatment

\section{Introduction}

In the last decade, the prevalence of overweight and obesity (defined as BMI $>25 \mathrm{~kg} / \mathrm{m}^{2}$ and $>30 \mathrm{~kg} / \mathrm{m}^{2}$, respectively) and their complications (especially type 2 diabetes, cardiovascular diseases, obstructive sleep apnea, osteoarthritis, male and female infertility, and certain forms of cancer) has greatly increased among adults and children. It has become an alarming medical and social issue ${ }^{1}$ resulting from socioeconomic and behavioral changes in modern society (leading to increased energy consumption and decreased energy expenditure), ${ }^{2}$ and biological factors. ${ }^{3}$

Weight loss of 5\%-10\% of initial body weight reduces cardiovascular and metabolic health risks associated with obesity. ${ }^{4}$ International Health Guidelines ${ }^{4,5}$ recommend lifestyle modification as the first strategy in the management of obesity. If lifestyle modification alone is ineffective, pharmacotherapy may be considered for individuals with a BMI $\geq 30 \mathrm{~kg} / \mathrm{m}^{2}$ or for those with a BMI $\geq 27 \mathrm{~kg} / \mathrm{m}^{2}$ with comorbidities (eg, hypertension, diabetes, obstructive sleep apnea, type 2 diabetes) or a family history of overweight. Bariatric surgery should be reserved for individuals with a BMI $\geq 40 \mathrm{~kg} / \mathrm{m}^{2}$ or $\geq 35 \mathrm{~kg} / \mathrm{m}^{2}$ and comorbidities who do not lose weight with lifestyle modification and pharmacotherapy. ${ }^{4,5}$ Very recently, US Food and Drug Administration has approved the use of Allergan's LAP-Band Adjustable Gastric Banding System for people with a BMI $>35 \mathrm{~kg} / \mathrm{m}^{2}$ without comorbidities or for $>30 \mathrm{~kg} / \mathrm{m}^{2}$ and at least one comorbidity. ${ }^{6}$
Correspondence: Federica Guaraldi Department of Pathology, The Johns Hopkins School of Medicine, 720 Rutland Avenue, 2I 205 Baltimore, MD, USA

$\mathrm{Tel}+$ I 443287891 I

Fax +I 410 6143548

Email fguaral I@jhmi.edu
Diabetes, Metabolic Syndrome and Obesity:Targets and Therapy 201 I:4 229-243

(c) 20II Guaraldi et al, publisher and licensee Dove Medical Press Ltd. This is an Open Access article which permits unrestricted noncommercial use, provided the original work is properly cited. 
Several studies have shown that the different antiobesity drugs, in conjunction with lifestyle treatments, can induce a weight loss up to $5 \%-10 \%$, even if there is a great variability in individual response to a specific treatment. ${ }^{7-9}$ Several different treatments, based on lifestyle modifications, ${ }^{10-12}$ drugs, ${ }^{13}$ and surgery ${ }^{14,15}$ have been developed in the past, and some others are currently being studied. ${ }^{13}$

The investigation for factors responsible for obesity onset and response to antiobesity treatments started in the early $1960 \mathrm{~s},{ }^{16,17}$ and the number of studies in this field has multiplied over the years. Most studies showed a wide variability in inter-individual response to all kinds of treatments, ${ }^{18}$ a usually modest result in weight loss and maintenance, ${ }^{19}$ and the involvement of many environmental, genetic, and behavioral factors. ${ }^{19-23}$ However, predictors of outcome continue to be poorly understood. Availability of good predictors would allow physicians to match treatment to patients, and would improve cost-efficacy and patient's chances of success in losing and maintaining weight. 22,23

This article focuses on factors that have been suggested as possible predictors of weight loss and/or weight maintenance in patients treated with orlistat, diethylpropion, mazindol, sibutramine, and topiramate (Table 1).

\section{Methods and study description}

A literature search was performed using PubMed database entering the drug name "and weight loss" for drugs approved by the FDA specifically for weight loss (orlistat, phentermine, and diethylpropion); drugs previously approved by the FDA that have been recently withdrawn from the market (sibutramine, mazindol, and rimonabant); and drugs approved by the FDA for other indications that exhibit weight loss promoting effects (topiramate, bupropion, and zonisamide). We found articles focusing on predictors in patients treated only with diethylpropion, orlistat, sibutramine, mazindol, and topiramate. Some studies investigated treatment outcomes in patients treated with an association of phentermine and fenfluoramine but, because this compound is no longer available and no comparative data with each single drug exists, these articles have not been included. Additional relevant articles from reference lists were included.

Only articles focusing on adults were included. Absolute or relative weight loss was the only considered variable. Thirty-three studies investigating gender, anthropometric, demographic, psychological, behavioral, and hereditary characteristics, associated medical conditions and habits,

Table I Main characteristics of antiobesity medications included in the study

\begin{tabular}{|c|c|c|c|c|}
\hline \multicolumn{5}{|c|}{ Drugs approved by FDA specifically for weight loss } \\
\hline Drug & Mechanism of action & Typical dosing & Adverse effects & Effect on weight \\
\hline Orlistat ${ }^{\mathrm{a}, \mathrm{d}, 9,33}$ & $\begin{array}{l}\text { Limits fats absorption; } \\
\text { pancreatic lipase inhibitor }\end{array}$ & $120 \mathrm{mg}$ tid & $\begin{array}{l}\text { Abdominal pain, } \\
\text { bloating, flatulence, oily stools, } \\
\text { diarrhea, decreased absorption } \\
\text { of fat soluble vitamins } \\
\text { Liver injury (rare) }\end{array}$ & $\begin{array}{l}2.9 \mathrm{~kg} \text { placebo subtracted } \\
\text { weight loss at I year }\end{array}$ \\
\hline Diethylpropion ${ }^{\mathrm{a}, \mathrm{e}, 9}$ & $\begin{array}{l}\text { Appetite suppressant; } \\
\text { sympathomimetic amine }\end{array}$ & Average $75 \mathrm{mg} / \mathrm{d}$ & $\begin{array}{l}\text { Dizziness, headache, } \\
\text { insomnia, restless, increase } \\
\text { in blood pressure, palpitations, } \\
\text { tachycardia, gastrointestinal } \\
\text { symptoms, rash }\end{array}$ & $\begin{array}{l}3.0 \mathrm{~kg} \text { placebo-subtracted } \\
\text { weight loss in studies } \\
\text { ranging } 6-52 \text { weeks }\end{array}$ \\
\hline Sibutramine $e^{\mathrm{b}, 9,33}$ & $\begin{array}{l}\text { Appetite suppressant; } \\
\text { serotonin and norepinephrine } \\
\text { reuptake inhibitor }\end{array}$ & $10-15 \mathrm{mg} / \mathrm{d}$ & $\begin{array}{l}\text { Hypertension, } \\
\text { tachycardia }\end{array}$ & $\begin{array}{l}4.2 \mathrm{~kg} \text { placebo-subtracted } \\
\text { weight loss at I year }\end{array}$ \\
\hline Mazindolb,13 & $\begin{array}{l}\text { Appetite suppressant; } \\
\text { norepinephrine inhibitor. } \\
\text { Inhibitor of gastric acids } \\
\text { and insulin secretion }\end{array}$ & $\mathrm{I}-4 \mathrm{mg} / \mathrm{d}$ & $\begin{array}{l}\text { Restlessness, hypertension, } \\
\text { nervousness }\end{array}$ & $\begin{array}{l}\text { Average } 3 \mathrm{~kg} \text { placebo subtracted } \\
\text { weight loss in } 12 \text { week studies }\end{array}$ \\
\hline Topiramate ${ }^{c, 33}$ & $\begin{array}{l}\text { Weight loss mechanism } \\
\text { unknown. Supposed } \\
\text { monoamine-mediated appetite } \\
\text { suppression; increase in fat } \\
\text { metabolism and reduction } \\
\text { in lipogenesis }\end{array}$ & $90-200 \mathrm{mg} / \mathrm{d}$ & $\begin{array}{l}\text { Cognitive impairment, } \\
\text { peripheral neuropathy }\end{array}$ & $\begin{array}{l}6.5 \% \text { pooled random-effects } \\
\text { of topiramate on weight loss } \\
\text { compared with placebo }\end{array}$ \\
\hline
\end{tabular}

Notes: aDrugs approved by FDA specifically for weight loss; 'Drugs previously approved by FDA specifically for weight loss and recently withdrawn from the market; 'Drugs approved by the FDA for the other indications that exhibit weight loss promoting effects; ${ }^{d}$ Drug approved by FDA for long-term treatment; ${ }^{\circ}$ Drug approved by FDA for short-term treatment ( 12 weeks). 
dietetic advices, and environmental factors were identified and included in this review.

All studies included subjects with a $\geq 25 \mathrm{~kg} / \mathrm{m}^{2}$ of both genders, except for two ${ }^{24,25}$ including females only. Age ranged from 16 to 70 years. The number of patients included in the different studies ranged from 36 to 3277 . The duration of the study varied from 8 to 208 weeks. Some included a run-in phase. ${ }^{26-31}$ The main characteristics of the patient sample and program of the considered studies are displayed in Table 2.

\section{Predictors associated with orlistat treatments}

Orlistat is a semisynthetic derivative of lipstatin which irreversibly and selectively binds to pancreatic lipases, reducing fat absorption by approximately $30 \%$ (Table 3 ). ${ }^{32}$ The prescribed dose is $120 \mathrm{mg}$, three times/day with meals. A meta-analysis ${ }^{33}$ including approximately 10,000 participants treated for at least 1 year, reported a mean placebo-subtracted weight loss of $2.9 \mathrm{~kg}$. Abdominal pain, bloating, flatulence, oily stools, and diarrhea represent common adverse effects; severe liver injury is rare. ${ }^{34}$

\section{Demographic variables}

Only one study ${ }^{35}$ investigated the impact of gender on weight loss, and found no significant difference in the percentage of initial body weight lost by males and females.

\section{Psychological factors and eating behaviors} Orlistat weight loss was positively related to the traits 'order' $(P<0.05)$, 'deliberation' $(P<0.01)$, and 'self-discipline', included in the main personality factor conscientiousness ${ }^{35}$ (which reflect the ability to have and to persist with a goal-directed and motivated behavior, hence to consider matters before acting, to be methodical, well organized, and self-disciplined). This suggests that people with a stronger personality and great conscientiousness can achieve higher levels of long-term compliance towards a high demanding treatment like orlistat, a characterized thrice-daily regimen, and dietary fat restriction. The importance of determination on treatment's results is evident in European prescribing information ${ }^{36}$ that, based on several observations, ${ }^{26}$ orlistat is only recommended to those patients who have previously lost at least $2.5 \mathrm{~kg}$ by diet alone in a 4-week period, as an indication of their capacity to comply with prescribed dietary changes. Conversely, levels of restrained eating, anxiety, and depression showed no impact on the final outcome. ${ }^{35}$

\section{Diabetes}

Two studies ${ }^{30,37}$ pointed out the difficulty in losing weight experienced by diabetic patients treated with orlistat.

\section{Initial weight loss}

A study conducted on 229 patients who completed a 2-year treatment ${ }^{26}$ showed that people who lost $\geq 5 \%$ of their initial weight in the first 12 weeks achieved better final results than people who did not $(-11.9 \pm 0.8 \%$ vs $-4.7 \pm 0.5 \%$; $P=0.0001)$. This finding was confirmed by another study ${ }^{38}$ in which $>80 \%$ of patients who achieved $\geq 5 \%$ reduction in their body weight after 3 and 6 months maintained $>5 \%$ weight loss and $>50 \%$ achieved $\geq 10 \%$ weight loss after 12 months $(P<0.01)$. Accordingly, European ${ }^{36}$ and National Institute for Clinical Excellence ${ }^{39}$ prescribing guidelines, which recommend that only people who lose $\geq 5 \%$ of initial body weight after 12 weeks and $\geq 10 \%$ after 6 months should continue taking orlistat.

\section{Associated dietetic and environmental factors}

Pharmacist's support was shown to improve patient's persistence with orlistat therapy but it did not improve the total amount of weight loss. ${ }^{40}$ The total daily calories assumed with diet (500 vs $1000 \mathrm{kcal} / \mathrm{day})^{38}$ did not have a marked impact on the overall clinical outcome of weight reduction (average weight loss 11.8 and $11.4 \mathrm{~kg}$ respectively), while a low fat, but also carbohydrate, consumption was essential to lose weight. ${ }^{31}$

\section{Predictors associated with diethylproprion treatments}

Diethylpropion is an amphetamine like analog currently approved by FDA for short-term weight loss. ${ }^{9}$ A metaanalysis conducted on several studies in which patients were treated with diethylpropion $75 \mathrm{mg} /$ day in combination with lifestyle treatment showed an average $3.0 \mathrm{~kg}$ of additional weight loss, compared with placebo. ${ }^{9}$ Common side effects include central nervous system stimulation, dizziness, headache, insomnia, restlessness, mild increases in blood pressure, palpitations, mild tachycardia, mild gastrointestinal symptoms, and rash. ${ }^{9}$

To our knowledge, only one study ${ }^{41}$ focused on predictors of weight loss in patients treated with diethylpropion, showing no statistically significant effect of patients' personality on weight loss, but just a positive correlation between social conformity and treatment completion $(P<0.004)$. 
Table 2 Main characteristics of the patient sample and program of the considered studies

\begin{tabular}{|c|c|c|c|}
\hline Author, year & Sample & Type of study & Program \\
\hline Alberici et $\mathrm{al}^{77}$ & $\begin{array}{l}\text { BL: } 5 \text { I p. } \\
\text { ET: } 49 \text { p. } \\
\text { BLCPCS: } \mathrm{mA} 44.1 \pm \text { I } .5 . \mathrm{F} 36 . \\
\text { mBMI } 24.0 \pm 3.4\end{array}$ & Prospective study & $\begin{array}{l}\text { Treatment:Topiramate was administered following } \\
\text { slow up titration; full dosage of } 100 \mathrm{mg} / \text { day, bid } \\
\text { regimen, reached within } 4 \text { weeks. } 4 \text { months treatment }\end{array}$ \\
\hline $\begin{array}{l}\text { Ben- } \\
\text { Menachem et } \mathrm{a}^{75}\end{array}$ & $\begin{array}{l}\text { BL: } 49 \text { p. F } 28 . \mathrm{mA} 36.2 \pm \text { II.7. } \mathrm{mBW} \\
77.8 \pm \text { 16.8. mBMI } 26.9 \pm 5.9 . \\
\text { ET: } 38 \text { p. F 22. mA } 35.9 \pm \text { II.5. } \\
\text { mBW } 75.8 \pm \text { I4.8. mBMI } 26.3 \pm 9.3 .\end{array}$ & Prospective study & $\begin{array}{l}\text { Treatment: I year. Topiramate added to pre-existing } \\
\text { anticonvulsant therapy. Starting dose } 25 \mathrm{mg} / \text { day, } \\
\text { increased biweekly in } 25 \text { or } 50 \mathrm{mg} \text { increments to the } \\
\text { best-tolerated dosage providing maximum seizure } \\
\text { control mean dosage after } 3 \text { months } 81 \mathrm{mg} / \text { day } \\
(21-154 \mathrm{mg} / \text { day })\end{array}$ \\
\hline Elfhag et $\mathrm{a}^{27}$ & $\begin{array}{l}\text { BL: } 36 \text { p. } \\
\text { ET: } 30 \text { p. } \\
\text { BLCPCS: F 22. mA } 43 \pm I 2(20-64) \\
\text { mBMI } 40 \pm 4(33-45)\end{array}$ & Prospective study & $\begin{array}{l}\text { Run in phase: } 6 \text { weeks. Clinical trial evaluating } \\
\text { the effect of sibutramine on food consumption in } \\
\text { laboratory test meals. } 2 \text { weeks each of: sibutramine, } \\
\text { placebo, and a wash out period (crossover design) } \\
\text { Treatment: } 26 \text { weeks. Sibutramine } \\
\text { ( } 15 \mathrm{mg} / \text { day) + monthly dietary advice }\end{array}$ \\
\hline $\begin{array}{l}\text { Elfhag and } \\
\text { Rossner }{ }^{19}\end{array}$ & $\begin{array}{l}\text { BL: } 36 \mathrm{p.} \\
\text { F 27. mA } 43 \pm 12 \text { years }(20-64) \\
\mathrm{mBMI} 39 \pm 4(30-45) \\
\text { ET: } 3 \text { I p. }\end{array}$ & Prospective study & $\begin{array}{l}\text { Run in phase: } 6 \text { weeks. Sibutramine/placebo and } \\
\text { wash out period Treatment: } 26 \text { weeks. } 15 \mathrm{mg} / \text { day } \\
\text { sibutramine and dietary advice in monthly group } \\
\text { sessions with a dietitian }\end{array}$ \\
\hline Elfhag et $\mathrm{a}^{35}$ & $\begin{array}{l}\text { BLCPCS: } 478 \text { p. } \\
\text { F } 30 I . m A 42.4 \pm 12.2(16-70) \\
\text { mBMI } 42.4 \pm \text { I } 2.2(26-68)\end{array}$ & $\begin{array}{l}\text { Retrospective analysis of } \\
\text { patients who completed } \\
\text { the study }\end{array}$ & $\begin{array}{l}\text { I. Weight Watcher treatment } \\
\text { (control) or } \\
\text { 2. Sibutramine or } \\
\text { 3. Orlistat }\end{array}$ \\
\hline Fabricatore et al ${ }^{45}$ & $\begin{array}{l}\text { BL: } 224 \text { P. } \\
\text { F I80. mA } 43.8 \pm 10.2 . \\
\text { C I } 46 . \text { OE } 76 . \\
\text { Sibutramine: } 55 \mathrm{p} . \\
\text { Lifestyle modification: } 55 \mathrm{p} . \\
\text { Combined therapy: } 60 \mathrm{p} . \\
\text { Sibutramine + brief behavioral } \\
\text { therapy: } 54 \mathrm{p} . \\
\text { ET: } 185 \mathrm{p} .\end{array}$ & Prospective study & $\begin{array}{l}52 \text { weeks treatment: } \\
\text { I200- } 1500 \text { kcal/day diet ( } 15 \% \text { proteins, } 30 \% \text { fats, } 55 \% \\
\text { carbohydrates) + walking + random assignment to: } \\
\text { a. Sibutramine: } 8 \text { visits of } 10-15 \text { minutes with a } \\
\text { primary care provider + pamphlet providing tips } \\
\text { for healthy lifestyle + sibutramine } 15 \mathrm{mg} / \text { day } \\
\text { b. Lifestyle modifications: } 30 \text { group behavior } \\
\text { modification sessions } \\
\text { c. Combined therapy (a }+ \text { b) } \\
\text { d. Sibutramine + brief therapy: sibutramine } \\
\quad 15 \mathrm{mg} / \text { day }+8 \text { sessions of } 10-15 \text { minutes behavior } \\
\text { therapy sessions }\end{array}$ \\
\hline Finer et $\mathrm{a}^{29}$ & $\begin{array}{l}\text { BL: } 928 \text { p. } \\
\text { Nondiabetic: } 771 \mathrm{p} . \\
\text { F 6II. C } 763 . \\
\text { mA } 42.4 \pm 10.7 . \\
\text { mBW } 97.5 \pm 15.2 . \\
\text { mBMI } 35.0 \pm 4.1 . \\
\text { Diabetic: I } 57 \text { p. } \\
\text { mA } 51.4 \pm 9.6 . \\
\text { F } 84 . \text { C I53. } \\
\text { mBW I0I.3 } \pm 17.7 . \\
\text { mBMI } 35.7 \pm 5.4 .\end{array}$ & $\begin{array}{l}\text { Retrospective analysis of } \\
\text { patients included in } \\
7 \text { different studies }\end{array}$ & $\begin{array}{l}\text { Run in phase: placebo run-in phase ( } 3 \text { studies); } \\
\text { open-label sibutramine run-in phase ( } 2 \text { studies); } \\
\text { very low calorie diet (I study) } \\
\text { Treatment: } 52 \text { weeks; random assignment to } \\
\text { sibutramine ( } 10 \mathrm{mg} / \text { day) or placebo ( } 6 \text { studies with } \\
\text { fixed dose; I study with dose titrated depending on } \\
\text { patient's weight maintenance) }\end{array}$ \\
\hline Frey et $\mathrm{al}^{50}$ & $\begin{array}{l}\text { BLCPCS: I I0 p. } \\
\text { C. F 74. A 42-48. } \\
\text { BMI 35.3-35.4. } \\
\text { Sibutramine group: } \\
54 \text { p. F 38. mA } 46.8 \pm 10.5 . \\
\text { mBW } 99.2 \pm 14.1 . \\
\text { mBMI } 35.1 \pm 4.2 . \\
\text { Placebo group: } 56 \text { p. } \\
\text { F } 36 . \mathrm{mA} 49.3 \pm 11.0 \\
\text { mBW } 104.5 \pm 15.2 . \\
\text { mBMI } 35.5 \pm 3.3 .\end{array}$ & $\begin{array}{l}\text { Retrospective study on a } \\
\text { part of patients who had } \\
\text { completed the program }\end{array}$ & $\begin{array}{l}54 \text { weeks treatment: random assignment to } \\
15 \mathrm{mg} / \text { day of sibutramine or placebo + } \\
\text { behavior program ( } 16 \text { group sessions) + } \\
\text { physical activity + diet (daily energy } \\
\text { requirement minus } 500 \text { to } 1000 \mathrm{kcal} / \text { day) }\end{array}$ \\
\hline
\end{tabular}


Table 2 (Continued)

\begin{tabular}{|c|c|c|c|}
\hline Author, year & Sample & Type of study & Program \\
\hline Greenway $^{78}$ & $\begin{array}{l}\text { SIBUTRAMINE: } 83 \text { obese } \\
\text { patients with diabetes; } \\
2004 \text { obese patients } \\
\text { without diabetes. } \\
\text { ORLISTAT: } 32 \text { I obese } \\
\text { patients with diabetes; } 2404 \text { obese } \\
\text { patients without diabetes. } \\
\text { MAZINDOL: } 40 \text { obese } \\
\text { patients with diabetes; } \\
998 \text { obese patients } \\
\text { without diabetes. }\end{array}$ & $\begin{array}{l}\text { Retrospective comparative } \\
\text { analysis between a study } \\
\text { focusing on diabetic } \\
\text { patients and an average } \\
\text { of studies in nondiabetic } \\
\text { patients for each } \\
\text { type of drug }\end{array}$ & $\begin{array}{l}12 \text { weeks sibutramine treatment or } \\
52 \text { weeks orlistat treatment or } \\
12 \text { weeks mazindol treatment }\end{array}$ \\
\hline Grudell et $a^{47}$ & 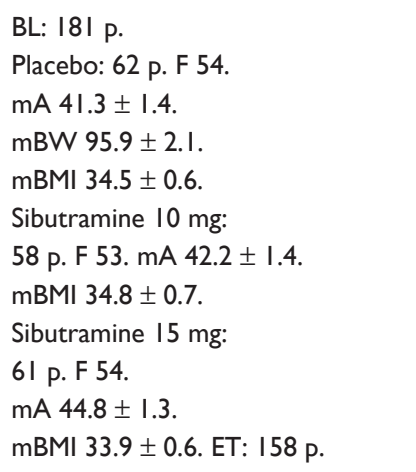 & Prospective study & $\begin{array}{l}\text { I } 2 \text { weeks treatment: behavioral therapy (LEARN } \\
\text { manual }+ \text { I group session of } 15 \text { minutes each week } \\
\text { led by a psychologist) + placebo or sibutramine } \\
10 \mathrm{mg} / \text { day or } 15 \mathrm{mg} / \text { day }\end{array}$ \\
\hline Hainer et $\mathrm{al}^{24}$ & $\begin{array}{l}\text { BL: } 80 \text { p. } \\
\text { F. } \mathrm{mA} 43.9 \pm 10.6 \text { (I8-65). } \\
\text { mBMI } 36.7 \pm 4.8 \text { (30-45). } \\
\text { Ist phase: sibutramine } 38 \mathrm{p} . \\
\text { Placebo } 42 \text { p. } 2 \text { nd phase: } 80 \mathrm{p} . \\
\text { ET: } 67 \text { p. }\end{array}$ & Prospective study & $\begin{array}{l}\text { Ist phase: } 4 \text { months; random assignment to } \\
\text { sibutramine } 10 \mathrm{mg} / \text { day or placebo } \\
\text { 2nd phase: } 8 \text { months; sibutramine }+5-6 \mathrm{MJ} / \text { day } \\
\text { diet ( } 50 \%-60 \% \text { carbohydrates; } 15 \%-20 \% \text { proteins; } \\
25 \%-30 \% \text { fat) + physical activity + food diary records }\end{array}$ \\
\hline Hansen et $\mathrm{al}^{46}$ & $\begin{array}{l}\text { BL: } 605 \mathrm{p} . \\
\text { BMI } 30-45 ; 18-65 \mathrm{yr} \\
\text { Completers of the } \\
\text { Ist phase: } 505 \mathrm{p} . \\
\mathrm{mA} 40.4 \pm 10.4 . \\
\mathrm{mBW} 102.5 \pm 15.3 . \\
\mathrm{mBMI} 36.7 \pm 4 . \mathrm{I} . \\
\text { Enrolled in the } \\
\text { 2nd phase: } 467 \mathrm{p} . \\
352 \text { sibutramine. } \\
\text { II } 5 \text { placebo. ET: } 263 \mathrm{p} .\end{array}$ & Prospective study & $\begin{array}{l}\text { Ist phase: } 6 \text { months; sibutramine ( } 10 \mathrm{mg} / \text { day) and } \\
\text { LCD (patient's estimated daily energy expenditure } \\
\text { minus } 600 \mathrm{kcal} / \text { day. } 45 \%-50 \% \text { carbohydrates; } \\
30 \% \text { fats; } 15 \%-20 \% \text { proteins) } \\
\text { 2nd phase: } 18 \text { months; random assignment for } \\
\text { patients who achieved } \geq 5 \% \text { of weight loss to } \\
\text { sibutramine } 10 \mathrm{mg} / \text { day ( } 352 \mathrm{p} \text { ) or placebo ( } 115 \mathrm{p})\end{array}$ \\
\hline Hauner et $\mathrm{al}^{53}$ & $\begin{array}{l}\text { BL: } 348 \text { p. BMI } 30-40 \text {; } 18-65 \text { yr. } \\
\text { Sibutramine: I } 74 \text { p. } \\
\text { F I36. mA } 44.5 \pm \text { I.2. mBW } \\
\text { I00.2 } \pm \text { I.9. mBMI } 35.6 \pm 0.6 . \\
\text { Placebo: I } 74 \text { p. F I } 23 . \\
\text { mA } 47.3 \pm \text { I.3. mBW } \\
\text { I05.4 } \pm \text { 2.0. mBMI } 35.7 \pm 0.5 .\end{array}$ & $\begin{array}{l}\text { Retrospective analysis } \\
\text { of patients included } \\
\text { in the study }\end{array}$ & $\begin{array}{l}54 \text { weeks treatment: } 20 \text { group sessions and } \\
\text { prescription of a diet ( } 500 / 1000 \mathrm{kcal} \text { minus } \\
\text { of the daily required energy expenditure) }+ \\
\text { physical activity + sibutramine or placebo } \\
\text { (random assignment) } \\
2 \text { years follow up }\end{array}$ \\
\hline Hsiao et $\mathrm{a}^{54}$ & $\begin{array}{l}\text { BL: I3I p. Sibutramine: } \\
87 \text { p. Placebo } 44 \text { p. } \\
\text { ET: I I } 8 \text { p. } \\
\text { BLCPCS: } \\
\text { Sibutramine } 82 \text { p. F } 4 \text { I. } \\
\text { mA } 31.7 \pm 4.9 \\
\text { mBW Placebo } 37 \text { p. F } 17 . \\
\text { mA } 31 . I \pm 5.8 \\
\text { mBW } 83.8 \pm 15.0 \\
\text { mBMI } 29.8 \pm 3.4\end{array}$ & Prospective study & $\begin{array}{l}12 \text { weeks treatment: } \\
\text { sibutramine } 10 \mathrm{mg} / \text { day or placebo }\end{array}$ \\
\hline
\end{tabular}

(Continued) 
Table 2 (Continued)

\begin{tabular}{|c|c|c|c|}
\hline Author, year & Sample & Type of study & Program \\
\hline Hsiao et $\mathrm{al}^{57}$ & $\begin{array}{l}\text { BL: I3I p. } \\
\text { Sibutramine: } 87 \text { p. } \\
\text { Placebo } 44 \text { p. } \\
\text { ET: I I } 8 \text { p. } \\
\text { BLCPCS: Sibutramine } 82 \text { p. F } 4 \text { I. } \\
\text { mA } 3 I .7 \pm 4.9 \\
\text { mBW Placebo } 37 \text { p. F I7. } \\
\text { mA } 3 I . I \pm 5.8 \\
\text { mBW } 83.8 \pm \text { I } 5.0 \\
\text { mBMI } 29.8 \pm 3.4\end{array}$ & Prospective study & $\begin{array}{l}12 \text { weeks treatment: } \\
\text { sibutramine } 10 \mathrm{mg} / \text { day or placebo }\end{array}$ \\
\hline Klein et $\mathrm{al}^{76}$ & $\begin{array}{l}\text { BL: } 26 \text { p. } \\
\text { ET: } 22 \text { p. F I3. BLCPCS: mA 4I.5. } \\
\text { mBMI } 28.0 \pm 6.6 \\
\text { mBW } 80.3 \pm 19.3\end{array}$ & Prospective study & 6 months treatment: topiramate \\
\hline $\begin{array}{l}\text { LLoret- } \\
\text { Linares et } \mathrm{al}^{37}\end{array}$ & $\begin{array}{l}\text { BL: } \\
\text { Sibutramine ( } 3 \text { studies): } \\
\text { I I5-467 p. F 76\%-84\%. } \\
\text { mA 40.4-43.3. mBMI 32.4-37.9. } \\
\text { Orlistat (I } 2 \text { studies): } \\
\text { 422-3277 p. F 55\%-87\%. } \\
\text { mA 40-53. mBMI 34.7-37.4. } \\
\text { Rimonabant (4 studies): } \\
688-1826 \text { p. }\end{array}$ & $\begin{array}{l}\text { Retrospective comparative } \\
\text { analysis between studies } \\
\text { considering nondiabetic } \\
\text { and diabetic subjects } \\
\text { for each type of drug }\end{array}$ & $\begin{array}{l}\text { Sibutramine: } 10 \text { or } 20 \mathrm{mg} \text { once a day for } \\
52 \text { or } 104 \text { weeks or } \\
\text { Orlistat: } 120 \mathrm{mg} \text { three times a day for } \\
\text { 52/76/104/208 weeks or } \\
\text { Rimonabant: } 20 \mathrm{mg} \text { daily for } 52 \text { or } 104 \text { weeks. All } \\
\text { trials combined pharmacological therapy with a } \\
\text { reduced-calorie diet }\end{array}$ \\
\hline $\begin{array}{l}\text { Malone and } \\
\text { Alger-Maye }^{40}\end{array}$ & $\begin{array}{l}\text { BL: } 30 \text { p. F } 26 . \\
\text { mA } 43.8 \pm 9.7 . \\
\text { mBW I } 27 \pm 34 . \\
\text { mBMI } 45.5 \pm \text { II.8. } \\
\text { Intervention group: I } 5 \text { p. } \\
\text { F I4. mA } 44.9 \pm 10.5 . \\
\text { BW I } 30 \pm 39 . \text { BMI } 48.3 \pm 14.6 . \\
\text { Control group: } \\
\text { F I2. mA } 42.8 \pm 9 . \\
\text { BW I } 24 \pm 30 . \text { BMI } 42.8 \pm 8.1 . \\
\text { ET: } 9 \text { p. Intervention group: } \\
7 \text { p. Control: } 2 \text { p. }\end{array}$ & Prospective study & $\begin{array}{l}\text { Patients randomly assigned to the group followed by } \\
\text { a community pharmacist (trained and educated for } \\
\text { I day to support clients during weight loss treatment } \\
\text { with orlistat) or to the group not followed by a } \\
\text { pharmacist and followed for } 26 \text { weeks }\end{array}$ \\
\hline Norris et $\mathrm{al}^{30}$ & $\begin{array}{l}\text { BL: } 3 \text { I studies. } \\
\text { mA 48-66. F 50\%. } \\
\text { Fluoxetine (6 studies): } \\
296 \text { p (total number). } \\
\text { Orlistat (8 studies): } \\
2036 \text { p (total number). } \\
\text { Sibutramine (8 studies): } \\
\text { I047 p (total number). } \\
\text { Cimetidine I study. } \\
\text { Diethylpropion (3 studies): } \\
40-58 \text { p (single study). } \\
\text { Mazindol (3 studies); } \\
\text { I0-64 p (single study). } \\
\text { Phentermine ( } 2 \text { studies). }\end{array}$ & $\begin{array}{l}\text { Meta-analysis of studies } \\
\text { considering nondiabetic } \\
\text { and diabetic subjects } \\
\text { for each type of drug }\end{array}$ & $\begin{array}{l}\text { Run-in period: I-5 weeks in most studies; } \\
\text { placebo + dietary counseling } \\
\text { Treatment: Fluoxetine: } 8 \text { to } 52 \text { weeks or } \\
\text { Orlistat: } 12 \text { to } 57 \text { weeks or } \\
\text { Sibutramine: } 12 \text { to } 52 \text { weeks or } \\
\text { Cimetidine: } 12 \text { weeks } \\
\text { Dyethylpropion: } 8 \text { to } 40 \text { weeks or } \\
\text { Mazindol: } 6 \text { to } 12 \text { weeks or } \\
\text { Phentermine: } 16 \text { to } 26 \text { weeks }\end{array}$ \\
\hline Peters et $\mathrm{al}^{25}$ & $\begin{array}{l}\text { BL: I49 p. F. mA } \\
54 \pm 5.78(45-65) \\
\text { mBW I03.4I } \pm 20.65 \\
(68.03-175.54) \\
\text { mBMI } 40.10 \pm 8.01(30-76) \\
\text { FU: } 74 \text { p. }\end{array}$ & Prospective study & $\begin{array}{l}6 \text { months treatment: sibutramine } \\
15 \mathrm{mg} / \text { day }+\mathrm{I} \text { hour/month behavior modification } \\
\text { seminar + daily exercise }\end{array}$ \\
\hline Rissanen et $\mathrm{al}^{26}$ & $\begin{array}{l}\text { BLCPCS: } 220 \text { p. } \\
\text { F I } 78 . \text { A } 42-49 . \\
\text { BW } 90.3-101.9 \\
\text { BMI 32.7-36.I. }\end{array}$ & $\begin{array}{l}\text { Retrospective analysis of } \\
\text { patients who completed } \\
\text { the treatment in two } \\
\text { different studies }\end{array}$ & $\begin{array}{l}\text { Run in phase: } 4 \text { weeks; hypocaloric diet ( } 30 \% \text { fat; } \\
500 \text { kcal/day energy deficit) + placebo } \\
\text { Treatment: } 2 \text { years; random assignment to: } \\
120 \mathrm{mg} / \text { day orlistat or placebo + diet }\end{array}$ \\
\hline
\end{tabular}


Table 2 (Continued)

\begin{tabular}{|c|c|c|c|}
\hline Author, year & Sample & Type of study & Program \\
\hline Risser et $\mathrm{al}^{63}$ & $\begin{array}{l}\text { BL: } \\
\text { PAR': } 22 \text { p. } \\
\text { F } 90.9 \% . \\
\text { mA } 49.9 \pm 12 . \\
\text { mBMI } 39.3 \pm 7 \\
\text { Non-PAR: } 47 \text { p. } \\
\text { F 78.7\%. mA } 47.1 \pm 10 \\
\text { mBMI } 37.1 \pm 6.12\end{array}$ & Retrospective analysis & $\begin{array}{l}\text { Ist phase: } 8 \text { weeks; } 800-1220 \mathrm{kcal} / \text { day diet + } \\
\text { 24-hour food diary + visits (first } 10 \text { weeks: } \\
\text { 2-5 days/week) + sibutramine } 15 \text { g/daily } \\
\text { (flexible doses adjusted depending on patient) } \\
\text { 2nd phase: } 40 \text { weeks; weight maintenance } \\
\text { through well-balanced meals and regular } \\
\text { exercise + sibutramine } 15 \mathrm{mg} / \text { day } \\
\text { (flexible doses adjusted depending } \\
\text { on patient) }\end{array}$ \\
\hline Rodin et $\mathrm{al}^{41}$ & $\begin{array}{l}\text { BL: } 204 \text { p. } \\
\text { Clinic I: I } 44 \text { p. } \\
\text { F I29. A I8-59. } \\
\text { BW } 28 \%-199 \% \text { overweight. } \\
\text { Clinic 2: } 60 \text { p. F } 60 \text {. A I8-54. } \\
\text { BW I7\%-I83\% overweight. } \\
\text { ET: clinic I: } 33 \text { p. } \\
\text { Clinic 2: } 56 \text { p. }\end{array}$ & Prospective study & $\begin{array}{l}\text { Clinic I: I } 4 \text { weeks ( } 8 \text { weeks treatment): random } \\
\text { assignment to } 4 \text { groups: behavior modification } \\
\text { treatment, diethylpropion } 65 \mathrm{mg} / \text { day, } \\
\text { mazindol } 2 \mathrm{mg} / \text { day, placebo } \\
\text { Clinic } 2 \text { : } 10 \text { weeks; ( } 9 \text { weeks treatment): behavior } \\
\text { therapy for all patients + random assignment to two } \\
\text { different formulations of mazindol or placebo }\end{array}$ \\
\hline Shimizu and Mori' ${ }^{66}$ & $\begin{array}{l}\text { BL: } 24 \text { p. Trp64Trp: } \\
\text { I6 p. Trp64Arg: } 8 \text { p. } \\
\text { BMI }>35 \mathrm{~kg} / \mathrm{m}^{2}\end{array}$ & Prospective study & $\begin{array}{l}\text { I } 2 \text { weeks treatment: mazindol (starting dose } \\
0.5 \mathrm{mg} / \text { day. Increments of } 0.5 \mathrm{mg} / \text { day every } \\
2 \text { weeks up to } 1.5 \mathrm{mg} / \text { day at week } 6 \text { and }\end{array}$ \\
\hline Shimizu et $\mathrm{al}^{67}$ & BL: 4 I p. F $25 . \mathrm{BMI}>35$. & & then continued until week I2) \\
\hline Tankova et al ${ }^{59}$ & 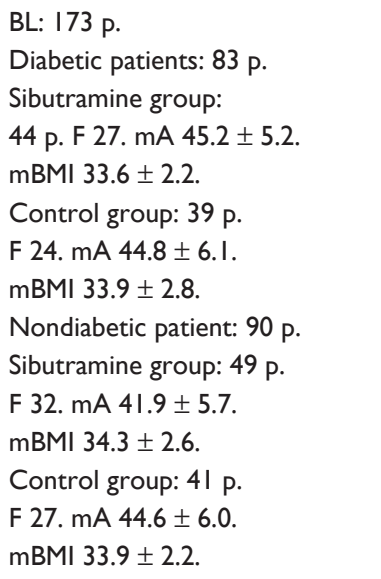 & Prospective study & $\begin{array}{l}3 \text { months treatment: hypocaloric diet ( } 600 \mathrm{kcal} / \\
\text { day deficit on the estimated total daily energy } \\
\text { expenditure. }<30 \% \text { fat, I } 5 \% \text { protein, } 55 \%-60 \% \\
\text { carbohydrates) }+30 \text { minutes walking/day + random } \\
\text { assignment to sibutramine once daily or placebo. } \\
\text { First month dosage of sibutramine was } 10 \mathrm{mg} / \mathrm{day} \\
\text { replaced by I } 5 \mathrm{mg} / \text { day in case of no change } \\
\text { in body weight }\end{array}$ \\
\hline Toplak et $\mathrm{al}^{38}$ & 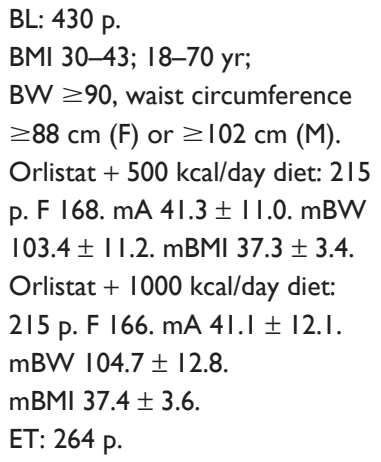 & Prospective study & $\begin{array}{l}\text { I year treatment: orlistat I } 20 \text { mg } 3 \text { times/ } \\
\text { day + dietary counseling + daily food diary + random } \\
\text { assignment to a diet ( } 50 \% \text { carbohydrates, } 30 \% \text { fat, } \\
20 \% \text { protein) of } 500 \text { or } 1000 \mathrm{kcal} / \text { day deficit } \\
\text { Patients who achieved a } 5 \% \text { weight loss at both } \\
\text { months } 3 \text { and } 6 \text { ( } 295 \text { p) were allowed to continue } \\
\text { the study until month I } 2\end{array}$ \\
\hline Theisen et $\mathrm{al}^{74}$ & $\begin{array}{l}\text { BL: } 26 \text { p. F I0. mA } \\
37.4 \pm 10.3(20.7-65.3) \\
\text { M mBMI } 28.1 \pm 6.4 \\
\text { F mBMI } 22.5 \pm 3.5 \\
\text { ET: I8 p. }\end{array}$ & Prospective study & $\begin{array}{l}25 \text { weeks treatment with topiramate } \\
\text { added to existing anticonvulsant therapy } \\
\text { (carbamazepine I } 2 \text { p; carmanazepine + valproate } \\
3 \text { p; carbamazepine + lamotrigine } 3 \text { p; } \\
\text { carbamazepine + valproate + lamotrigine } 3 \text { p; } \\
\text { carbamazepine + valproate + phenobarbital I p; } \\
\text { carbamazepine + phenobarbital I p; } \\
\text { carbamazepine + phenytoin I p; } \\
\text { phenytoin + phenobarbital I p; } \\
\text { phenytoin + lamotrigine + phenobarbital I p) }\end{array}$ \\
\hline
\end{tabular}

(Continued) 
Table 2 (Continued)

\begin{tabular}{|c|c|c|c|}
\hline Author, year & Sample & Type of study & Program \\
\hline Ullrich et $\mathrm{al}^{31}$ & $\begin{array}{l}\text { BL: } 26 \mathrm{I} \text { p. F } 2 \mathrm{I} 4 \\
\mathrm{~mA} 4 \mathrm{I} .4 \pm 9.8 \\
\text { mBMI } 36.8 \pm 4.2 \\
\text { Sibutramine group: } 204 \mathrm{p} . \\
\text { F I68. } \mathrm{mA} 4 \mathrm{I} .4 \pm 9.7 \\
\text { mBMI } 36.6 \pm 4.2 \\
\text { Placebo group: } 57 \mathrm{p} . \\
\text { F } 46 . \mathrm{mA} 4 \mathrm{I} .3 \pm 10.0 \\
\mathrm{mBMI} 37 . \mathrm{I} \pm 3.8\end{array}$ & $\begin{array}{l}\text { Retrospective analysis of } \\
\text { people who completed the } \\
\text { study }\end{array}$ & $\begin{array}{l}\text { Run in phase: } 4 \text { weeks; hypocaloric diet ( } 600 \mathrm{kcal} / \\
\text { day deficit on patient's estimated basal metabolic } \\
\text { rate. } \leq 30 \% \text { fat; protein and carbohydrate ad } \\
\text { libitum) + placebo ( } 3 \text { times/day with meals) } \\
\text { Treatment: } 72 \text { weeks; orlistat ( } 120 \mathrm{mg} \times 3 / \text { day). } \\
\text { 4-day food diary at weeks }-4,-2,0,12,24,36,48 \text {, } \\
64 \text {. Group weight management program sessions } \\
\text { (one each } 4 \text { weeks) }\end{array}$ \\
\hline Van Baak et al ${ }^{48}$ & $\begin{array}{l}\text { BL: } 605 \text { p. BMI } 30-45 ; 17-65 \text { yr. } \\
\text { After } 6 \text { mo run in phase: } 467 \text { p. } \\
\text { ET: } 26 I \text { p. F } 2 \text { I } 4 . \text { mA } 4 I .4 \pm 9.8 \text {. } \\
\text { BMI } 36.8 \pm 4.2\end{array}$ & Prospective study & $\begin{array}{l}\text { Ist phase: } 6 \text { months; sibutramine ( } 10 \mathrm{mg} / \text { day) and } \\
\text { LCD (patient's estimated daily energy expenditure } \\
\text { minus } 600 \mathrm{kcal} / \text { day. } 45 \%-50 \% \text { carbohydrates; } 30 \% \\
\text { fats; I } 5 \%-20 \% \text { proteins) } \\
\text { 2nd phase: I } 8 \text { months; random assignment for } \\
\text { patients who achieved } \geq 5 \% \text { of weight loss to } \\
\text { sibutramine } 10 \mathrm{mg} / \text { day ( } 352 \mathrm{p} \text { ) or placebo (II } 5 \text { ). } \\
\text { Dietary advice and one meeting with a } \\
\text { dietician a month }\end{array}$ \\
\hline $\begin{array}{l}\text { Vazquez } \\
\text { Roque et al }{ }^{44}\end{array}$ & $\begin{array}{l}\text { BL: } 48 \text { p. Sibutramine } 25 \text { p. } \\
\text { Placebo } 23 \text { p. } \\
\text { Overweight: } 24 \text { p. } \\
\text { mA } 36.7 \pm \text { I.7. F I7. C } 87.5 \% \\
\text { mBW } 79.6 \pm \text { I.6. } \\
\text { mBMI } 27.9 \pm 0.3 \\
\text { Obese: } 24 \text { p. mA } \\
44.2 \pm 2.6 . \text { F I } 3 . \\
\text { C } 95.8 \% \text { mBW } 98.2 \pm 2.7 \\
\text { mBMI } 34.8 \pm 0.7 . \\
\text { ET: } 43 \text { p. Sibutramine } 22 \text { p. } \\
\text { Placebo } 2 \text { I p. }\end{array}$ & Prospective study & $\begin{array}{l}\text { I } 2 \text { weeks treatment: LEARN manual (self-help } \\
\text { behavioral manual for weight loss) }+10-15 \text { minutes } \\
\text { behavior therapy sessions (one every } \\
4 \text { weeks) + random assignment to sibutramine } \\
\text { I5 mg/day or placebo }\end{array}$ \\
\hline Womble et $\mathrm{al}^{65}$ & $\begin{array}{l}\text { BL: Group I: } 59 \text { p. F } 3 \mathrm{I} \\
\text { M: } \mathrm{mA} 50.2 \mathrm{I} \pm 7.39 \\
\mathrm{mBMI} 39.94 \pm 5.99 \\
\mathrm{~F}: \mathrm{mA} 45.72 \pm 9.2 \\
\mathrm{mBMI} 40.34 \pm 8.20 \\
\text { Group } 2: 32 \text { p taken } \\
\text { from group I. F I } 5 . \\
\text { ET group I: } 59 \mathrm{p} . \\
\text { Group } 2: 32 \text { p. }\end{array}$ & Prospective study & $\begin{array}{l}6 \text { months treatment for group I; } 12 \text { months } \\
\text { treatment for group } 2 \\
\text { For both groups, random assignment to: } \\
\text { a. Fenfluramine + mazindol ( } 6 \text { months; } \\
29 \text { p. } 12 \text { months; I } 8 \text { p) or } \\
\text { b. Fenfluramine + phentermine ( } 6 \text { months; } \\
25 \text { p. } 12 \text { months; II p) or } \\
\text { c. Caffeine + ephedrine ( } 6 \text { months; } 2 \text { p. } 12 \text { months, I p) or } \\
\text { d. Mazindol }(6 \text { months, } 3 \text { p. } 12 \text { months, } 2 \text { p) }\end{array}$ \\
\hline
\end{tabular}

Notes: 'Cases: patients who had received a prescription for sibutramine in response to priori authorization through their health care insurer (PAR). Controls: subjects who did not receive reimbursement, although they were prescribed, sibutramine (non-PAR).

Abbreviations: BL, sample characteristics at base line; BMI, body mass index $\left(\mathrm{kg} / \mathrm{m}^{2}\right)$; BLCPCS, baseline characteristics of people who completed the study; BW, body weight; C, Caucasians; F, number of female patients; $\mathrm{ET}$, sample characteristics at the end of the program; $\mathrm{mA}$, mean age; $\mathrm{mBMI}$, mean body mass index $\left(\mathrm{kg} / \mathrm{m}^{2}\right)$; $\mathrm{mBW}$, mean body weight; OE, other ethnicity; p, number of patients; yr, years old; LCD, low-calorie diet.

\section{Predictors associated with sibutramine treatments}

Sibutramine is a centrally-acting drug that reduces energy intake and increases satiety mainly through the inhibition of serotonine and norepinephrine reuptake (Table 4). ${ }^{8}$ Weight loss is enhanced by the stimulation of thermogenesis ${ }^{42,43}$ and the delay in gastric emptying. ${ }^{44}$ The prescribing dose was $10-15 \mathrm{mg}$ once daily. A recent meta-analysis $\mathrm{s}^{33}$ that included 2,838 participants treated with sibutramine for at least 1 year, showed a mean placebo-subtracted weight loss of $4.2 \mathrm{~kg}$.
The drug was withdrawn from the market in October 2010 because of cardiovascular side effects. ${ }^{13}$

\section{Psychological factors and eating behaviors} A study in $2003^{27}$ demonstrated that patients with more deviating levels (elevated or lowered from the mean) of physical demand states (inborn and natural primary drives, including hunger) and dependency orientation (which implies preoccupation with oral activity such as eating and dependency on food, and which is typical of 
Table 3 Predictors of weight loss and maintenance in patients treated with orlistat

\begin{tabular}{|c|c|c|}
\hline & Author, year & Predictors \\
\hline Demographic and anthropometric factors & Elfhag et $\mathrm{al}^{35}$ & PPWL: male gender \\
\hline Psychological factors and eating behaviors & Elfhag et $\mathrm{al}^{35}$ & $\begin{array}{l}\text { PPWL: high levels of order and deliberation } \\
\text { (conscientiousness). High levels of self-discipline } \\
\text { and conscientiousness ntPWL: high levels of eating } \\
\text { restraint, neuroticism, anxiety, and depression }\end{array}$ \\
\hline $\begin{array}{l}\text { Initial weight loss in the lead-in period } \\
\text { and under treatment }\end{array}$ & Rissanen et $\mathrm{al}^{26}$ & $\begin{array}{l}\text { PPWL: Weight loss } \geq 2.5 \mathrm{~kg} \text { during } 4 \text { weeks lead-in } \\
\text { period; weight loss } \geq 5 \% \text { body weight after } 12 \text { weeks } \\
\text { treatment; } \geq 10 \% \text { body weight after } 6 \text { months }\end{array}$ \\
\hline & Toplak et al ${ }^{38}$ & PPWL: $\geq 5 \%$ weight loss at 3 and 6 months \\
\hline $\begin{array}{l}\text { Associated medical, dietetic, } \\
\text { and environmental factors }\end{array}$ & $\begin{array}{l}\text { Malone and Alger-Mayer }{ }^{40} \\
\text { Toplak et } \mathrm{al}^{38} \\
\text { Ullrich et } \mathrm{al}^{31}\end{array}$ & $\begin{array}{l}\text { ntPWL: pharmacist's support } \\
\text { ntPWL: } 500 \mathrm{kc} / \text { day diet vs } 1000 \mathrm{kcal} / \text { day diet } \\
\text { PPWL: low fat and carbohydrate intake }\end{array}$ \\
\hline
\end{tabular}

Abbreviations: NPWL, negative predictor of weight loss; NPWM, negative predictor of weight maintenance; ntPWL, not predictor of weight loss; ntPWM, not predictor of weight maintenance; PPWL, positive predictor of weight loss; PPWM, positive predictor of weight maintenance.

people vulnerable to social isolation) lost more weight when treated with sibutramine $(r=0.533, P=0.002$ and $r=0.478, P=0.008$, respectively). Initial signs of difficulties concerning physical demand states were strong positive predictors of weight loss $(r=0.533, P=0.002)$, explaining $27 \%$ alone. These findings suggest that the drug could improve conscious control over instinctual drives; alternatively, it could increase the sensitivity to alterations in hunger and satiety, helping people with initial high levels of hunger urges to improve control on food intake. The enhanced satiety effect of sibutramine could also have helped patients with high oral dependency to give less importance to food and the support provided by being enrolled in a treatment program could have helped them to abstain from food.

In the same study, ${ }^{27}$ self-inspect ability (which means an ability to monitor and reflect on one's behavior and thinking) was related to weight loss but was not a predictor.

Several studies ${ }^{24,28,35}$ have found that patients with more unrestrained eating (less cognitive control and conscious determination to resist eating, less strategic dieting) achieved greater weight loss, suggesting a negative influence of a pre-trial weight suppression on the subsequent treatment with sibutramine. ${ }^{24}$ At the same time, the increase in dietary restraint and the decrease in disinhibition score during weight reduction phase guaranteed the success of weight loss maintenance. ${ }^{24}$

Discordant results were obtained about the role of depression on weight loss. Two studies ${ }^{27,28}$ pointed out no correlations between depression and weight loss; two found a negative association between depression and weight loss; $;^{24,45}$ one $^{35}$ showed depression as a positive predictor $(r=0.40$, $P<0.05)$.

\section{Demographic and anthropometric factors}

Except for one study that pointed out a positive association between younger age and weight loss, ${ }^{35}$ age, $, 27,45,46$ and gender ${ }^{27,46}$ appeared to be unrelated to the ability to lose weight. According to the only study that considered the influence of race on weight loss, ${ }^{45}$ Caucasian ethnicity appeared a positive predictor (odds ratio [OR]: $0.42, P=0.003$ ). Two authors ${ }^{35,46}$ speculated that patients with higher pre-treatment body weight could achieve greater weight loss $(r=0.27$, $P<0.001)$ because of their higher energy expenditure (resting metabolic rate) $(r=0.13, P=0.003) .{ }^{46}$ Conversely, another study ${ }^{27}$ found no association between initial weight and weight loss at 6 months $(r=-0.220, P=0.242)$.

\section{Familial obesity and personal weight history}

One study ${ }^{27}$ pointed out the positive effect that the absence of familial obesity and having been normal weight at some points as an adult had on weight loss, even if these factors were not statistically selected as predictors (Student's $t$-value $=3.239, P=0.003$; Student's $t$-value $=2.194$, $P=0.037$ respectively). Conversely, Fabricatore et al ${ }^{45}$ obtained better results treating patients with a longer history of overweight. Hansen et $\mathrm{al}^{46}$ showed the number of previous slimming attempts and the age of onset of obesity had no influence on final weight loss.

\section{Initial weight loss under treatment}

Several studies ${ }^{24,27,29,45-48}$ showed that early adherence to the program, early weight loss in the lead-in period and in the first 4 weeks of treatment were the strongest positive predictors of weight loss and maintenance, both in diabetic and nondiabetic patients. Accordingly, sibutramine prescribing guidelines ${ }^{49}$ 
Table 4 Predictors of weight loss and maintenance in patients treated with sibutramine

\begin{tabular}{|c|c|c|}
\hline & Author, year & Predictors \\
\hline \multirow[t]{8}{*}{ Psychosocial factors and eating behaviors } & Elfhag et $\mathrm{al}^{27}$ & $\begin{array}{l}\text { PPWL: high deviating levels of demand states (hunger); high levels of } \\
\text { dependency orientation and oral traits. Self-inspective ability } \\
\text { ntPWL: levels of anxiety, depression, bodily concern; eating disorders; } \\
\text { affective response to external stimuli }\end{array}$ \\
\hline & Elfhag and & PPWL: unrestrained eating (less cognitive control and conscious determination \\
\hline & Rossnerl ${ }^{19}$ & to resist from eating, less strategic dieting) \\
\hline & & ntPWL: hunger and disinhibition score; depressive features \\
\hline & Elfhag et $\mathrm{a}^{35}$ & $\begin{array}{l}\text { PPWL: unrestrained eating (emotional and external eating); high levels of } \\
\text { neuroticism, anxiety and depression. High levels of self-consciousness and hostility }{ }^{2}\end{array}$ \\
\hline & Fabricatore et $\mathrm{al}^{45}$ & NPWL: depressive symptoms \\
\hline & Hainer et $\mathrm{al}^{24}$ & NPWL: high dietary restraint at baseline; high initial depression score \\
\hline & & $\begin{array}{l}\text { PPWL: increase in dietary restraint during first months of treatment PPWM: } \\
\text { decrease in disinhibition scores }\end{array}$ \\
\hline \multirow[t]{5}{*}{ Demographic and anthropometric factors } & Elfhag et $\mathrm{al}^{27}$ & ntPWL: age; gender; pretreatment body weight \\
\hline & Elfhag et a ${ }^{35}$ & PPWL: young age \\
\hline & Fabricatore et $\mathrm{al}^{45}$ & PPWL: Caucasian ethnicity ntPWL: age \\
\hline & Hansen et $\mathrm{al}^{46}$ & PPWL: high baseline body weight (high resting metabolic rate) \\
\hline & & ntPWL: age; gender \\
\hline \multirow[t]{3}{*}{ Familial obesity and personal weight history } & Elfhag et $\mathrm{a}^{27}$ & NP: familial history of obesity; being obese during all adulthood \\
\hline & Fabricatore et $\mathrm{al}^{45}$ & PPWL: having been overweight for a long time \\
\hline & Hansen et $\mathrm{al}^{46}$ & ntPWL: age of obesity onset; number of previous slimming attempts \\
\hline Initial weight loss in the run in period & Elfhag et $\mathrm{a}^{27}$ & PPWL: great initial weight loss \\
\hline \multirow{8}{*}{ and under treatment } & Fabricatore et $\mathrm{al}^{45}$ & PPWL: early adherence and weight loss \\
\hline & Finer et $\mathrm{al}^{29}$ & PPWL: high early weight loss ( $\geq 4 \mathrm{~kg}$ at 3 months) \\
\hline & Hainer et $\mathrm{a}^{24}$ & PPWL: high initial weight loss \\
\hline & Hansen et $\mathrm{al}^{46}$ & PPWL: high initial weight loss in the run-in period; high initial weight \\
\hline & & loss under treatment \\
\hline & & ntPWL: number of previous slimming attempts; age of onset of obesity \\
\hline & van Baak et al ${ }^{48}$ & PPWM: high initial weight loss \\
\hline & Grudell et al ${ }^{47}$ & PPWL: high initial (4 weeks) weight loss \\
\hline \multirow[t]{9}{*}{ Genetic factors } & Frey et $\mathrm{al}^{50}$ & PPWL: A allele carriers position - 1211 of GNAS gene promoter \\
\hline & Grudell et $\mathrm{al}^{47}$ & $\begin{array}{l}\text { PPWL: gene pairs } 5 \text { HTTLPR LS/SS and GN } \beta 3 \text { GN } \beta 3 \text { rs5443 TC/TT; } \\
\alpha 2 \mathrm{~A} C \mathrm{C} \text { and GN } \beta 3 \text { rs5443 TC/TT }\end{array}$ \\
\hline & Hauner et $\mathrm{al}^{53}$ & PPWL and PPWM: CC genotype of GN $\beta 3$ rs 5443 \\
\hline & Hsiao et al ${ }^{54}$ & PPWL: T allele carriers (TT or TC genotype) of GN $\beta 3$ rs 5443 \\
\hline & Hsiao et $\mathrm{a}^{57}$ & PPWL: -866 A polymorphism of UCP2; association between -866A \\
\hline & & polymorphism of UCP2 and rs $5443 \mathrm{~T}$ polymorphism of GN $\beta 3$ \\
\hline & Peters et $\mathrm{a}^{25}$ & PPWL: homozygous AI48A or GI48G polymorphism of PNMT \\
\hline & Vazquez & PPWL: LS/SS genotype of SLC6A4 ntPWL: $\alpha 2 A-129 \mid$ C/G; \\
\hline & Roque et a $\mathrm{a}^{44}$ & PNMT GI48A polymorphism; GN $\beta 3$ genotypes \\
\hline \multirow[t]{15}{*}{$\begin{array}{l}\text { Associated pathologies, habits, nutritional, } \\
\text { and environmental factors }\end{array}$} & Fabricatore et $\mathrm{al}^{45}$ & $\begin{array}{l}\text { PPWL: combined therapy (sibutramine + behavioral therapy) } \\
\text { ntPWL: distance from clinic }\end{array}$ \\
\hline & Finer et $\mathrm{a}^{29}$ & NPWL: diabetes \\
\hline & Greenway $^{78}$ & NPWL: diabetes \\
\hline & Hainer et $\mathrm{a}^{24}$ & PPWM: increase in protein intake; decrease in fat intake \\
\hline & & ntPWM: 8 or 12 months of treatment \\
\hline & Hansen et $\mathrm{al}^{46}$ & ntPWL: smoking history \\
\hline & LLoret- & NPWL: diabetes \\
\hline & Linares et $\mathrm{al}^{37}$ & \\
\hline & Norris et $\mathrm{al}^{30}$ & NPWL: diabetes \\
\hline & Peters et $\mathrm{al}^{25}$ & ntPWL: postmenopausal state, hysterectomy, hormone replacement. \\
\hline & & Fiber intake, total caloric intake, total fat intake \\
\hline & Risser et $\mathrm{al}^{63}$ & $\begin{array}{l}\text { PPWL: priori authorization to have sibutramine as a prescription partially } \\
\text { reimbursed (PAR) }\end{array}$ \\
\hline & Tankova et al ${ }^{59}$ & NPWL: diabetes \\
\hline & van Baak et al ${ }^{48}$ & PPWM: high levels of leisure-time physical activity \\
\hline & & ntPWM: macronutrient composition of the diet during weight maintenance period \\
\hline
\end{tabular}

Abbreviations: NPWL, negative predictor of weight loss; NPWM, negative predictor of weight maintenance; ntPWL, not predictor of weight loss; ntPWM, not predictor of weight maintenance; PPWL, positive predictor of weight loss; PPWM, positive predictor of weight maintenance. 
recommend physicians to increase the dose to $15 \mathrm{mg} /$ day or discontinue treatment in patients who have not lost $2 \mathrm{~kg}$ in the first 4 weeks of treatment with the dose of $10 \mathrm{mg}$, and to avoid treatment beyond 12 weeks in people who fail to lose $5 \%$ of their initial body weight.

\section{Genetic factors}

Some authors focused on the effect that several genetic polymorphisms could have on weight loss and maintenance. Frey et $\mathrm{al}^{50}$ investigated the role of the $\mathrm{G}>\mathrm{A}$ transition at position -1211 of the human $\mathrm{G}$ protein $\alpha$-subunit gene $(G N A S)$ promoter, showing that sibutramine was effective only in A carriers $(P=0.002)$ (GG genotypes showed no additional weight loss under sibutramine compared to placebo). $G$ protein $\alpha$-subunit is responsible for hormones coupling to the enzyme adenylyl-cyclase and is required in adipocytes for the lipolytic response to $\beta$ adrenergic agonists. $^{50}$

Four studies investigated GNB3, which encodes for the G $\beta 3$ subunit of heteromeric $G$ proteins (coupled to specific receptors, including serotonine and norepinephrine), key components of intracellular signal transduction, with a prominent role in body weight regulation. C825T (rs5443) polymorphism on exon 10 reduces lipolytic response to cathecolamines in fat cells $\mathrm{s}^{51,52}$ and is associated with obesity, hypertension, and diabetes. Hauner et $\mathrm{al}^{53}$ showed that sibutramine treatment was more effective in individuals with the $\mathrm{CC}$ genotype than in subjects with the TT/TC genotypes (weight loss: $7.2 \pm 2.2$ vs $4.1 \pm 2.1 \mathrm{~kg}, P=0.0013$ ). In the CC group, the OR for a weight loss $>5 \%$ (sibutramine vs placebo) was $6.6(P=0.004)$ and for a weight loss $>10 \%$ was $9.6(P=0.010)$. This was in contrast with two studies ${ }^{47,54}$ showing that patients with TT/TC genotypes achieved better results than $\mathrm{CC}$ genotype $(P=0.018$ and $P<0.001$ respectively), and another study ${ }^{44}$ pointing out no association between GN $\beta 3$ polymorphisms and weight loss.

One study ${ }^{25}$ focused on the positive association $(P=0.003)$ between weight loss and the homozygous variant (either G/G or A/A) G148A of PNMT (phenylethanolamine $\mathrm{N}$-methyltransferase) encoding for an adrenal enzyme catalyzing the conversion of norepinephrine to epinephrine ${ }^{55}$ but, in a second study, this result was not confirmed. ${ }^{44}$

Vazquez-Roque et $\mathrm{al}^{44}$ investigated also the role of "short" $(\mathrm{S})$ and "long" (L) repeats in the 5-HTT-linked region of the promoter of gene SLC6A4 (solute carrier family 6, member 4; chromosome 17q11.1-q12) encoding for the soluble serotonin transporter ${ }^{56}$ and pointed out the positive effect of SLC6A4 LS/SS genotype on weight loss (subjects with SLC6A4 LS/SS genotype had an average weight loss of $6.1 \pm 1.0 \mathrm{~kg}$, compared with a $0.1 \pm 0.9 \mathrm{~kg}$ average weight increase with placebo; subject with SLC6A4 LL genotype showed an average weight loss of $3.3 \pm 1.8 \mathrm{~kg}$ on placebo, compared with $3.9 \pm 1.6 \mathrm{~kg}$ weight loss in sibutramine group).

Grudell et $\mathrm{al}^{47}$ found the positive effect of the association of TC/TT genotype of C825T polymorphism of GN 33 gene and the CC genotype of $\alpha 2 \mathrm{~A}$ adrenoreceptor C1291G polymorphism or the 5HTTLPR LS/SS genotype.

Very recently, Hsiao et $\mathrm{al}^{57}$ used the same patients in which they had investigated the role of GN 33 polymorphism ${ }^{54}$ to study the role of $-866 \mathrm{G} / \mathrm{A}$ polymorphism on weight loss in patients treated with sibutramine. The uncoupling protein 2 (UCP2) gene encodes a mitochondrial transporter protein, which is highly expressed in adipose tissue, skeletal muscle, and pancreatic islets. ${ }^{58} \mathrm{~A}$ strong effect of sibutramine on weight loss was observed in individuals with the AA, GA, and AA + GA genotypes $(P=0.019, P<0.001$, and $P<0.001$, respectively) whereas the drug caused no significant effect in individuals with the GG genotype $(P=0.063)$. For the combination of UCP2 AA + GA with GN 33 TT + TC (carriers of at least one variant allele for each polymorphism), the $P$-value for treatment effects on weight loss was $<0.001$. In contrast, no effects were observed in patients with the wild type genotype combination of UCP2 GG and GN 33 . A significant $(P<0.001)$ gene interaction between UCP2 and GN 33 was identified by a 2-locus model.

\section{Associated pathologies, habits, nutritional, and environmental factors}

Diabetes represents the chronic disease that most often is associated with obesity. Weight loss improves glycemic control and can be achieved using pharmacological therapy. ${ }^{30,37,59}$ However in diabetic patients weight management is more challenging, in part because of the weight-promoting effects of the majority of anti-diabetic drugs ${ }^{37}$ and, on average, is achieved slower and less (up to 69\%) than in nondiabetic patients. ${ }^{29,30,37,59}$ This final aspect is recognized by the Australian regulatory authorities who suggest to withdraw sibutramine after 3 months in nondiabetic patients who have failed to lose $5 \%$ of their initial body weight but after 6 months in diabetic patients. ${ }^{60}$

On the other side, estrogen levels (ie, menopause, hormone replacement), hysterectomy, ${ }^{25}$ and smoking habit ${ }^{46}$ were demonstrated to not have a significant effect on weight loss in response to sibutramine.

The addition of behavioral therapy ${ }^{45}$ or high leisuretime physical activity ${ }^{48}$ to sibutramine showed a strong 
favorable effect on weight loss and maintenance, while dietetic modifications in fat, protein, and fiber intake $\mathrm{e}^{24,25}$ during the treatment, and the choice of 8 vs 12 months period of treatment ${ }^{24}$ did not have a great impact on outcomes. This is in accordance with most of studies on antiobesity drugs that demonstrate that the maximum weight loss is achieved after 6-8 months, whereas after 12-16 months of treatment, minor weight regain usually occurs. ${ }^{61,62}$

A priori authorization to have sibutramine partially reimbursed improved patients' compliance and weight loss. ${ }^{63}$ Distance to the clinic was marginally related to treatment success. $^{45}$

\section{Predictors associated with mazindol treatments}

Mazindol is a centrally acting drug that blocks the reuptake of norepinephrine by presynaptic neurons, increasing norepinephrine levels within the synaptic cleft (Table 5). The result is the stimulation of the $\beta 2$ adrenergic receptors in the lateral hypothalamus and the inhibition of feeding. ${ }^{11}$ It also inhibits gastric acid and insulin secretion, and increases locomotor activity. ${ }^{64}$

\section{Psychosocial factors and eating behavior}

A study in $1977^{41}$ found that high social conformity and emotional stability, extraversion, and need for social acceptance improved patients' adhesion to the program, whereas no personality characteristic predicted weight loss.

According to a more recent study, ${ }^{65}$ high levels of hunger, anxiety, and dietary restraint, and small perceived body size had a negative influence on weight loss in patients treated with mazindol. Suggested explanations for these findings were the drug incapability of controlling extreme levels of hunger, the limited slimming possibility in patients dieting before entering the treatment, and the individual lacking perception of the need for weight loss.

\section{Genetic factors}

Two studies ${ }^{66,67}$ pointed out a significantly greater body weight reduction in subjects with the Trp64 Trp allele than in those with Trp64Arg allele of ADR 33 gene (encoding for a protein which regulates cathecolamine-induced lipolysis ${ }^{68}$ mainly expressed in white adipose tissue) $(6.9 \pm 1.0 \mathrm{~kg}$ vs $3.6 \pm 0.7 \mathrm{~kg}$ in the first study and $8.4 \pm 1.7 \mathrm{~kg}$ vs $3.7 \pm 0.8 \mathrm{~kg}$ in the second study; $P<0.05)$.

\section{Predictors associated with topiramate treatment}

Topiramate is an anticonvulsant drug acting on sodium and calcium T-type channels (Table 6). The way in which it could promote weight loss is still unknown. Some authors have shown an increase in extraneuronal levels of dopamine, norepinephrine, and serotonin in the hippocampus and have speculated that a similar effect in the hypopthalamus could explain topiramate action as an appetite suppressor. ${ }^{69}$ Other studies have demonstrated an increase in fat metabolism (through the increased activity of lipoprotein lipase in adipose tissue and promotion of hepatic fat metabolism and decreased fatty acid synthesis) ${ }^{70,71}$ and the inhibition of lipogenesis through the inhibition of carbonic anhydrase II and $\mathrm{V}^{72}$

A recent meta-analysis ${ }^{9}$ based on studies conducted on epileptic patients reported an average placebo-subtracted weight loss of $6.5 \%$ in topiramate-treated patients $(P<0.001)$. Adverse effects are paresthesia, changes in taste, dizziness, memory impairment, insomnia, and somnolence..$^{9,73}$

\section{Demographic and anthropometric factors}

Three studies ${ }^{74-76}$ pointed out a correlation between higher initial BMI and a greater weight loss $(P<0.001)$ in epileptic patients treated with topiramate for a period of 10 weeks to 6 months. Only one study ${ }^{77}$ conducted on migraine patients treated for 4 months found no correlation between initial BMI, gender, and total weight loss $(P=0.44)$.

Table 5 Predictors of weight loss and maintenance in patients treated with mazindol

\begin{tabular}{|c|c|c|}
\hline & Author, year & Predictors \\
\hline \multirow{5}{*}{$\begin{array}{l}\text { Psychosocial factors and } \\
\text { eating behaviors }\end{array}$} & Rodin et $\mathrm{a}^{41}$ & PPWL: high levels of social conformity; belief that poor eating habits are crucial in producing obesity \\
\hline & & NPWL: high levels of responsiveness to external, non-food relevant stimuli; belief that hereditary \\
\hline & & or physical factors are causes of primary importance in producing overweight \\
\hline & & ntPWL: self-esteem and self-concept; emotional stability; extraversion; internal locus of control. \\
\hline & Womble et $a^{65}$ & NPWL: high levels of dietary restraint, perceived hunger and trait anxiety; small perceived body size \\
\hline \multirow[t]{3}{*}{ Genetic predictors } & Shimizu and & NPWL: Trp64Arg mutation of GNß3 gene (vs Trp64Trp) \\
\hline & Mori & \\
\hline & Shimizu et $\mathrm{al}^{67}$ & \\
\hline
\end{tabular}

Abbreviations: NPWL, negative predictor of weight loss; NPWM, negative predictor of weight maintenance; ntPWL, not predictor of weight loss; ntPWM, not predictor of weight maintenance; PPWL, positive predictor of weight loss; PPWM, positive predictor of weight maintenance. 
Table 6 Predictors of weight loss and maintenance in patients treated with topiramate

\begin{tabular}{|c|c|c|}
\hline & Author, year & Predictors \\
\hline Demographic and & Alberici et $\mathrm{al}^{77}$ & ntPWL: gender; initial BMI \\
\hline anthropometric & Theisen et $\mathrm{al}^{74}$ & PPWL: high initial BMI \\
\hline \multirow[t]{2}{*}{ factors } & Ben-Menachem et $\mathrm{al}^{75}$ & PPWL: high initial BMI \\
\hline & Klein et $\mathrm{al}^{76}$ & $\begin{array}{l}\text { PPWL: high initial BMI } \\
\text { and body fat }\end{array}$ \\
\hline Eating behavior & Klein et $\mathrm{a}^{76}$ & $\begin{array}{l}\text { PPWL: reduction in } \\
\text { hunger at } 3 \text { months }\end{array}$ \\
\hline
\end{tabular}

Abbreviations: ntPWL, not predictor of weight loss; PPWL, positive predictor of weight loss.

\section{Eating behavior}

One study ${ }^{76}$ reported a positive $(P=0.002)$ association between a reduction in hunger after 3 months of treatment and total weight loss at month 6 .

\section{Results}

Our analysis pointed out the overall paucity of reliable predictors of weight loss and maintenance associated with pharmacological antiobesity treatments, a characteristic previously described by studies focusing on lifestyle modification treatments. ${ }^{18,19}$

Indeed, among all considered factors, the early response to treatment was demonstrated to be a sure positive predictor of weight loss and maintenance. Conversely, diabetes appeared to have a strong negative impact on patients' ability to lose weight when treated with the different drugs.

A more unrestrained eating at baseline and high levels of determination and self-discipline appeared to be positive predictors of outcome in patients treated with sibutramine and orlistat, respectively, even if the statistical association was less strong.

For all other considered variables, published data are not sufficient to define their role on final outcomes.

\section{Conclusion}

Limitations related both to the disease and to the intervention program could explain these inconclusive and often conflicting results. Indeed, the multitude of factors involved in obesity's development and weight loss, and their complex interactions make each patient different from the other and the investigation of reliable predictors particularly challenging. Furthermore psycho-behavioral difficulties experienced by patients when starting a new treatment, and the lack of significant results often associated with the different therapies, are responsible for the high levels of attrition of patients from the weight-loss programs.

At the same time, our analysis pointed out important limitations related to currently available literature investigating outcomes associated to antiobesity pharmacological treatments. First of all, few studies have been conducted with the primary aim to point out the role of specific factors on weight loss and maintenance. Second, a minor part of these studies have included a large cohort of patients. Third, studies are very heterogeneous from the point of view of the number and characteristics of patients included, the duration and type of program, and the tests and parameters used for patients' evaluation. Finally, very often, the analysis and interpretation of the achieved results are very difficult and the power of the associations is reduced because of the limited number of subjects included.

Probably, the enrollment of a greater number of patients in future trials, the use of common protocols of treatment and parameters (for each category of predictors) to assess final outcomes could improve statistical significance of results and the ability to compare data. Moreover, because both obesity onset and weight loss are dependent on the interaction of a great number of different factors, it could be important to test different categories of predictors on the same group of patients.

Despite the above mentioned difficulties and the discouraging results obtained until recently, we believe that, because of obesity's social and medical impact and the availability of new promising antiobesity drugs (whose mechanisms of action are very often similar to those of old drugs; the most promising one is the association of bupropion and naltrexone), ${ }^{79}$ the search for reliable predictors of outcome associated to antiobesity treatments has to be supported and encouraged.

Being able to find reliable predictors of successful outcome for pharmacological treatments would mean to give prescribing physicians, patients, and the authorities a tool for antiobesity drug management.

\section{Disclosure}

No conflicts of interest were declared in relation to this paper.

\section{References}

1. James WP. The epidemiology of obesity: the size of the problem. J Intern Med. 2008;263(4):336-352.

2. Stein CJ, Colditz GA. The epidemic of obesity. J Clin Endocrinol Metab. 2004;89(6):2522-2525.

3. Rankinen T, Zuberi A, Chagnon YC, et al. The human obesity gene map: the 2005 update. Obesity (Silver Spring). 2006;14(4):529-644. 
4. World Health Organization. Obesity: preventing and managing the global epidemic. Geneva, Switzerland: Report of a WHO consultation. Technical Report Series 894. 2000.

5. Tsigos C, Hainer V, Basdevant A, et al. Management of obesity in adults: European clinical practice guidelines. Obes Facts. 2008;1(2): $106-116$.

6. US Food and Drug Administration News Release. FDA expands use of banding system for weight loss. Available at: http://www.fda. gov/NewsEvents/Newsroom/PressAnnouncements/ucm245617.htm. Accessed 23 May, 2011.

7. Halpern A, Mancini MC. Treatment of obesity: an update on anti-obesity medications. Obes Rev. 2003;4(1):25-42.

8. Ioannides-Demos LL, Proietto J, McNeil JJ. Pharmacotherapy for obesity. Drugs. 2005;65(10):1391-1418.

9. Li Z, Maglione M, Tu W, et al. Meta-analysis: pharmacologic treatment of obesity. Ann Intern Med. 2005;142(7):532-546.

10. Dubnov-Raz G, Berry EM. The dietary treatment of obesity. Endocrinol Metab Clin North Am. 2008;37(4):873-886.

11. Clifton PM. Dietary treatment for obesity. Nat Clin Pract Gastroenterol Hepatol. 2008;5(12):672-681.

12. Sodlerlund A, Fischer A, Johansson T. Physical activity, diet and behaviour modification in the treatment of overweight and obese adults: a systematic review. Perspect Public Health. 2009;129(3):132-142.

13. Ioannides-Demos LL, Piccenna L, McNeil JJ. Pharmacotherapies for obesity: past, current, and future therapies. J Obes. 2011;2011:179674.

14. Leff DR, Heath D. Surgery for obesity in adulthood. BMJ. 2009; 339:b3402.

15. Baker MK, Byrne TK, Feldmann ME. Surgical treatment of obesity. Prim Care. 2009;36(2):417-427.

16. Seaton DA, Rose K. Defaulters from a weight reduction clinic. J Chronic Dis. 1965;18(10):1007-1011.

17. Silverstone JT, Solomon T. Psychiatric and somatic factors in the treatment of obesity. J Psychosom Res. 1965;9(3):249-255.

18. Teixeira PJ, Going SB, Sardinha LB, Lohman TG. A review of psychosocial pre-treatment predictors of weight control. Obes Rev. 2005;6(1):43-65.

19. Elfhag K, Rossner S. Who succeeds in maintaining weight loss? A conceptual review of factors associated with weight loss maintenance and weight regain. Obes Rev. 2005;6(1):67-85.

20. Moreno-Aliaga MJ, Santos JL, Marti A, Martinez JA. Does weight loss prognosis depend on genetic make-up? Obes Rev. 2005;6(2): 155-168.

21. Herpertz S, Kielmann R, Wolf AM, Hebebrand J, Senf W. Do psychosocial variables predict weight loss or mental health after obesity surgery? A systematic review. Obes Res. 2004;12(10):1554-1569.

22. Kral JG. Selection of patients for anti-obesity surgery. Int J Obes Relat Metab Disord. 2001;25(Suppl 1):S107-S112.

23. Brownell KD. Behavioral, psychological, and environmental predictors of obesity and success at weight reduction. Int J Obes. 1984;8(5):543-550.

24. Hainer V, Kunesova M, Bellisle F, Hill M, Braunerova R, Wagenknecht M. Psychobehavioral and nutritional predictors of weight loss in obese women treated with sibutramine. Int J Obes (Lond). 2005; 29(2):208-216.

25. Peters WR, MacMurry JP, Walker J, Giese RJ Jr, Comings DE. Phenylethanolamine N-methyltransferase G-148A genetic variant and weight loss in obese women. Obes Res. 2003;11(3):415-419.

26. Rissanen A, Lean M, Rossner S, Segal KR, Sjostrom L. Predictive value of early weight loss in obesity management with orlistat: an evidence-based assessment of prescribing guidelines. Int J Obes Relat Metab Disord. 2003;27(1):103-109.

27. Elfhag K, Rossner S, Carlsson AM, Barkeling B. Sibutramine treatment in obesity: predictors of weight loss including rorschach personality data. Obes Res. 2003;11(11):1391-1399.

28. Elfhag K, Rossner S, Barkeling B, Rooth P. Sibutramine treatment in obesity: initial eating behaviour in relation to weight loss results and changes in mood. Pharmacol Res. 2005;51(2):159-163.
29. Finer N, Ryan DH, Renz CL, Hewkin AC. Prediction of response to sibutramine therapy in obese non-diabetic and diabetic patients. Diabetes Obes Metab. 2006;8(2):206-213.

30. Norris SL, Zhang X, Avenell A, Gregg E, Schmid CH, Lau J. Pharmacotherapy for weight loss in adults with type 2 diabetes mellitus. Cochrane Database Syst Rev. 2005;(1):CD004096.

31. Ullrich A, Erdmann J, Margraf J, Schusdziarra V. Impact of carbohydrate and fat intake on weight-reducing efficacy of orlistat. Aliment Pharmacol Ther. 2003;17(8):1007-1013.

32. Guerciolini R. Mode of action of orlistat. Int J Obes Relat Metab Disord. 1997;21(Suppl 3):S12-S23.

33. Rucker D, Padwal R, Li SK, Curioni C, Lau DC. Long term pharmacotherapy for obesity and overweight: updated meta-analysis. $B M J$. 2007;335(7631):1194-1199.

34. US Food and Drug Administration [FDA]. Early communication about an ongoing safety review orlistat (marketed as Alli and Xenical). FDA: Silver Spring, MD. 2011.

35. Elfhag K, Finer N, Rossner S. Who will lose weight on sibutramine and orlistat? Psychological correlates for treatment success. Diabetes Obes Metab. 2008;10(6):498-505.

36. European Agency for Evaluation of Medical Products.Committee for Proprietary Medicinal Products European Public Assessment Report (EPAR): Xenical. Available at: http:/www.ema.europa.eu/docs/en_GB/ document_library/EPAR_-_Product_Information/human/000154/ WC500058428.pdf. 2011.

37. Lloret-Linares C, Greenfield JR, Czernichow S. Effect of weightreducing agents on glycaemic parameters and progression to type 2 diabetes: a review. Diabet Med. 2008;25(10):1142-1450.

38. Toplak H, Ziegler O, Keller U, et al. X-PERT: weight reduction with orlistat in obese subjects receiving a mildly or moderately reducedenergy diet: early response to treatment predicts weight maintenance. Diabetes Obes Metab. 2005;7(6):699-708.

39. National Institute for Clinical Excellence (NICE) guidance on the prevention, identification, assessment and management of overweight and obesity in adults and children. Available at: http://www.nice.org. uk/nicemedia/live/11000/30365/30365.pdf. 2006.

40. Malone M, Alger-Mayer SA. Pharmacist intervention enhances adherence to orlistat therapy. Ann Pharmacother. 2003;37(11): 1598-1602.

41. Rodin J, Bray GA, Atkinson RL, et al. Predictors of successful weight loss in an outpatient obesity clinic. Int J Obes. 1977;1(1):79-87.

42. Lean ME. How does sibutramine work? Int J Obes Relat Metab Disord. 2001;25(Suppl 4):S8-S11.

43. Hansen DL, Toubro S, Stock MJ, Macdonald IA, Astrup A. The effect of sibutramine on energy expenditure and appetite during chronic treatment without dietary restriction. Int J Obes Relat Metab Disord. 1999;23(10):1016-1024.

44. Vazquez Roque MI, Camilleri M, Clark MM, et al. Alteration of gastric functions and candidate genes associated with weight reduction in response to sibutramine. Clin Gastroenterol Hepatol. 2007;5(7):829-837.

45. Fabricatore AN, Wadden TA, Moore RH, Butryn ML, Heymsfield SB, Nguyen AM. Predictors of attrition and weight loss success: results from a randomized controlled trial. Behav Res Ther. 2009;47(8): 685-691.

46. Hansen D, Astrup A, Toubro S, et al. Predictors of weight loss and maintenance during 2 years of treatment by sibutramine in obesity. Results from the European multi-centre STORM trial. Sibutramine Trial of Obesity Reduction and Maintenance. Int J Obes Relat Metab Disord. 2001;25(4):496-501.

47. Grudell AB, Sweetser S, Camilleri M, et al. A controlled pharmacogenetic trial of sibutramine on weight loss and body composition in obese or overweight adults. Gastroenterology. 2008;135(4):1142-1154.

48. van Baak MA, van ME, Astrup AV, et al. Leisure-time activity is an important determinant of long-term weight maintenance after weight loss in the Sibutramine Trial on Obesity Reduction and Maintenance (STORM trial). Am J Clin Nutr. 2003;78(2):209-214. 
49. US and European prescribing information. Abbott Laboratories. 2010.

50. Frey UH, Hauner H, Jockel KH, Manthey I, Brockmeyer N, Siffert W. A novel promoter polymorphism in the human gene GNAS affects binding of transcription factor upstream stimulatory factor 1, Galphas protein expression and body weight regulation. Pharmacogenet Genomics. 2008;18(2):141-151.

51. Danoviz ME, Pereira AC, Mill JG, Krieger JE. Hypertension, obesity and GNB 3 gene variants. Clin Exp Pharmacol Physiol. 2006;33(3): $248-252$.

52. Ryden M, Faulds G, Hoffstedt J, Wennlund A, Arner P. Effect of the (C825T) Gbeta(3) polymorphism on adrenoceptor-mediated lipolysis in human fat cells. Diabetes. 2002;51(5):1601-1608.

53. Hauner H, Meier M, Jockel KH, Frey UH, Siffert W. Prediction of successful weight reduction under sibutramine therapy through genotyping of the G-protein beta3 subunit gene (GNB3) C825T polymorphism. Pharmacogenetics. 2003;13(8):453-459.

54. Hsiao DJ, Wu LS, Huang SY, Lin E. Weight loss and body fat reduction under sibutramine therapy in obesity with the $\mathrm{C} 825 \mathrm{~T}$ polymorphism in the GNB3 gene. Pharmacogenet Genomics. 2009;19(9):730-733.

55. Wong DL, Lesage A, Siddall B, Funder JW. Glucocorticoid regulation of phenylethanolamine N-methyltransferase in vivo. FASEB J. 1992; 6(14):3310-3315.

56. Heils A, Teufel A, Petri S, et al. Allelic variation of human serotonin transporter gene expression. J Neurochem. 1996;66(6):2621-2624.

57. Hsiao TJ, Wu LS, Hwang Y, Huang SY, Lin E. Effect of the common $-866 \mathrm{G} / \mathrm{A}$ polymorphism of the uncopling protein 2 gene on weight loss and body composition under sibutramine therapy in an obese Taiwanese population. Mol Diagn Ther. 2010;14(2):101-106.

58. Dalgaard LT, Pedersen O. Uncoupling proteins: functional characteristics and role in the pathogenesis of obesity and type II diabetes. Diabetologia. 2001;44(8):946-965.

59. Tankova T, Dakovska G, Lazarova M, Dakovska L, Kirilov G, Koev D. Sibutramine in the treatment of obesity in type 2 diabetic patients and in nondiabetic subjects. Acta Diabetol. 2004;41(4):146-153.

60. Reductil (sibutramine hydrochloride) Australian prescribing information. Abbott Australasia Pty Ltd. March 5, 2004.

61. James WP, Astrup A, Finer N, et al. Effect of sibutramine on weight maintenance after weight loss: a randomised trial. STORM Study Group. Sibutramine Trial of Obesity Reduction and Maintenance. Lancet. 2000;356(9248):2119-2125.

62. Sjostrom L, Rissanen A, Andersen T, et al. Randomised placebocontrolled trial of orlistat for weight loss and prevention of weight regain in obese patients. European Multicentre Orlistat Study Group. Lancet. 1998;352(9123):167-172.

63. Risser JA, Vash PD, Nieto L. Does prior authorization of sibutramine improve medication compliance or weight loss? Obes Res. 2005;13(1): 86-92.

64. Inoue S. Clinical studies with mazindol. Obes Res. 1995;(3 Suppl 4): 549S-552S.

65. Womble LG, Williamson DA, Greenway FL, Redmann SM. Psychological and behavioral predictors of weight loss during drug treatment for obesity. Int J Obes Relat Metab Disord. 2001;25(3):340-345.
66. Shimizu H, Mori M. Trp64Arg mutation of beta- 2 adrenergic receptor gene determines weight losing effect of mazindol. Obes Res. 2004; 12(Suppl):A37.

67. Shimizu H, Tsuchiya T, Oh-I S, Ohtani K-I, Okada S, Mori M. Incidence of beta3-adrenergic receptor polymorphism and prediction of successful weight reduction with mazindol therapy in severely obese Japanese subjects. Obes Res Clin Pract. 2007;1:119-123.

68. Schiffelers SL, Saris WH, Boomsma F, van Baak MA. beta(1)- and beta(2)-Adrenoceptor-mediated thermogenesis and lipid utilization in obese and lean men. J Clin Endocrinol Metab. 2001;86(5): 2191-2199.

69. Yamamura S, Hamaguchi T, Ohoyama K, et al. Topiramate and zonisamide prevent paradoxical intoxication induced by carbamazepine and phenytoin. Epilepsy Res. 2009;84:172-186.

70. Richard D, Ferland J, Lalonde J, Samson P, Deshaies Y. Influence of topiramate in the regulation of energy balance. Nutrition. 2000;16: 961-966.

71. Liang Y, She P, Wang X, Demarest K. The messenger RNA profiles in liver, hypothalamus, white adipose tissue and skeletal muscle of female Zucker diabetic fatty rats after topiramate treatment. Metabolism. 2006;55:1411-1419.

72. De Simone G, Di Fiore A, Supuran CT. Are carbonic anhydrase inhibitors suitable for obtaining antiobesity drugs? Curr Pharm Des. 2008; $14: 655-660$.

73. Rosenstock J, Hollander P, Gadde K, Sun X, Strauss R, Leung A. A randomised, doubleblind, placebo-controlled, multicenter study to assess the efficacy and safety of topiramate controlled release in the treatment of obese type 2 diabetic patients. Diab Care. 2007;30: $1480-1486$.

74. Theisen FM, Beyenburg S, Gebhardt S, et al. A prospective study of body weight and serum leptin levels in patients treated with topiramate. Clin Neuropharmacol. 2008;31(4):226-230.

75. Ben-Menachem E, Axelsen M, Johanson EH, Stagge A, Smith U. Predictors of weight loss in adults with topiramate-treated epilepsy. Obes Res. 2003;11(4):556-562.

76. Klein KM, Theisen F, Knake S, et al. Topiramate, nutrition and weight change: a prospective study. $J$ Neurol Neurosurg Psychiatry. 2008;79(590):593.

77. Alberici A, Borroni B, Manelli F, et al. Topiramate weight loss in migraine patients. J Neurol Sci. 2009;278(1-2):64-65.

78. Greenway F. Obesity medications and the treatment of type 2 diabetes. Diabetes Technol Ther. 1999;1(3):277-287.

79. Greenway FL, Fujioka K, Plodkowski RA, et al; COR-I Study Group. Effect of naltrexone plus bupropion on weight loss in overweight and obese adults (COR-I): a multicentre, randomised, double-blind, placebocontrolled, phase 3 trial. Lancet. 2010;376(9741):595-605. Erratum in: Lancet. 2010;376(9741):594. Lancet. 2010;376(9750):1392.

\section{Publish your work in this journal}

Diabetes, Metabolic Syndrome and Obesity: Targets and Therapy is an international, peer-reviewed open-access journal committed to the rapid publication of the latest laboratory and clinical findings in the fields of diabetes, metabolic syndrome and obesity research. Original research, review, case reports, hypothesis formation, expert opinion and commentaries are all considered for publication. The manuscript management system is completely online and includes a very quick and fair peer-review system, which is all easy to use. Visit http://www.dovepress.com/testimonials.php to read real quotes from published authors. 